BOLETIN DE LA SOCIEDAD GEOLOGICA MEXJCANA

V. UII, (2000) 59-71

http://www.igeofcu.unam.mx/sgml

\title{
Evolución tectonomagmática post-paleocénica de la Sierra Madre Occidental y de la porción meridional de la provincia tectónica de Cuencas y Sierras, México
}

\author{
José Jorge Aranda-Gómez ${ }^{1}$ \\ Christopher D. Henry2 \\ James F. Luhr ${ }^{3}$ \\ 1 UNICIT, Instituto de Geología, UNAM, Apartado postal 1-742, Ouerétaro, Oro. 76001 México, \\ jjag@servidor.unam.mx \\ 2Nevada Bureau of Mines and Geology. University of Nevada, Reno. Reno, Nevada \\ 89557-008, USA, chenry@unr.edu \\ 3Department of Mineral Sciences, Smithsonian Institution, Washington, D.C. 20560. \\ jluhr@volcano.sLedu
}

\section{Resumen}

A partir del Eoceno, los eventos geológicos más importantes en la región han sido volcanismo y fal/amiento. La actividad magmática asociada a la subducción dio origen a la extensa y voluminosa Sierra Madre Occidental (SMO). Conforme cesó la subducción gradualmente, inició el magmatismo intraplaca en la región situada al este de la SMO y una combinación de volcanismo alcalino y toleítico en la región del Golfo de California.

El fallamiento normal formó el extremo meridional de la provincia tectónica de Cuencas y Sierras. Sobre parte de esta provincia se sobrepuso la extensión oblicua que generó la Provincia Extensional del Golfo.

En muchos sitios el volcanismo y fal/amiento fueron simultáneos o el inicio del fal/amiento siguió después de un lapso breve al del volcanismo. Ambos fenómenos sucedieron en toda la región en al menos cuatro pulsos de actividad: 32-27 Ma, 24-20 Ma, 12-10 Ma y < 5 Ma. Sin embargo, la intensidad y carácter de estos pulsos variaron de manera sistemática de un lugar a otro. Así, el frente del volcanismo voluminoso asociado a subducción y el fal/amiento normal intenso se desplazaron de NE a SW y fueron reemplazados hacia el interior del continente por actividad alcalina intraplaca y por extensión de menor magnitud.

Los magmas dominantemente félsicos de la SMO pudieron generarse por fusión parcial de la corteza, o por cristalización fraccionada de magmas provenientes del manto y asimilación simultánea de material cortical. Las edades isotópicas de zircones en xenolitos corticales de Chihuahua indican que del Eoceno al Oligoceno medio hubo un pulso térmico que coincidió con el volcanismo de la SMO y que a partir del Mioceno existen en la parte media y profunda de la corteza condiciones de P.T congruentes con metamorfismo de grado alto. El origen de estas perturbaciones térmicas pudo estar asociado, respectivamente, a la actividad magmática de la SMOy al volcanismo intraplaca acompañado por extensión cortical.

Los pulsos de actividad tectonomagmática coinciden en el tiempo con etapas en que 'se incrementó notablemente la tasa de divergencia entre las placas Farallón y Pacífica y/o con períodos de reorganización mayor de las placas en la costa del Pacífico. Esta actividad se vio reflejada en el interior del continente. 
Por ejemplo, en el intervalo -30-24 Ma hubo un incremento en la tasa de divergencia que causó volcanismo y extensión en la SMo. Por otro lado, coincidiendo con la formación del Protogolfo de California (-12 Ma), al este de la SMO, hubo extensión y volcanismo alcalino. En ese mismo período la orientación del frente volcánico de la SMO giró gradualmente de NW-SE a $\sim E-W$, y el carácter dominante de los magmas extravasados cambió de félsico a intermedio, estableciéndose la Faja Volcánica Transmexicana, el cual ha conservado esa orientación desde el Mioceno medio (-12Ma), aunque el frente volcánico se ha desplazado hacia el sur.

\section{Abstract}

Since the Eocene, the most important geologic events in the region were volcanism and faulting. Magmatism related to subduction generated the voluminous Sierra Madre Occidental (SMO). Coincident with the waning of subduction, intraplate-type magmatism began in the region east of the SMO, and a combination of alkaline and tholeiitic magmatism began in the Gulf of California region.

Normal faulting produced the southern extension of the Basin and Range tectonic province. Upon part of this province was superimposed the oblique extension that generated the Gulf Extensional Province.

In many places, volcanism and faulting were simultaneous, or the faulting began a short time after the initiation of volcanic activity. Available information suggests that both phenomena occurred throughout the region during at least four distinct pulses: 32-27 Ma, 24-20 Ma, 12-10 Ma, and <5 Ma. However, the character and intensity of these pulses varied in a systematic way from one site to another. For example. the front of the voluminous felsic-intermediate, subduction-related volcanism and intense normal faulting shifted from NE to SW and was replaced by intraplate-type alkalic volcanism and extension of lesser magnitude.

The dominant felsic magmas associated with the Sierra Madre Occidental (SMO) could have been generated by partial melting of the crust, or by crystal fractionation of mantle-derived melts and simultaneous assimilation of crustal materials. The isotopic ages of zircons in crustal xenoliths from Chihuahua record an Eocene to middle-Oligocene thermal pulse that appears to be related to SMO activity. These xenoliths also provide evidence for high-grade metamorphic conditions in the middle and lower crust since the Miocene, which may reflect heating associated with extension-related intraplate magmatism.

The observed pulses of tectonomagmatic activity correspond to periods of increased divergence rate between the Farallon and Pacific plates, and/or with times of major plate reorganization. These events along the Pacific Coast had a manifestation within the continental interior. For example, there was an increase of the divergence rate during the period -30-24 Ma that caused volcanism and extension in the SMo. Likewise, coincident with the opening of the Protogulf of California (-12 Ma), extension and intraplate-type volcanism occurred east of the SMo. About the same time the orientation of the SMO volcanic front changed from NW$S E$ to - E-W, and the composition of the erupted magmas changed from dominantly felsic to intermediate, marking the start of activity in the Trans Mexican Volcanic Belt (TMVB). The orientation of the TMVB has remained constant since the middle Miocene (-12 Ma), but the volcanic front has shifted toward the south.

\section{INTRODUCCIÓN}

Por su magnitud y duración, el volcanismo y faIlamiento son los eventos geológicos más importantes que han afectado a la región noroccidental de México, al este del Golfo de California (Figura 1a), a partir del Eoceno. La provincia volcánica de la Sierra Madre Occidental (SMO) es la acumulación más grande de ignimbritas silícicas hasta ahora conocida en la Tierra. Sus afloramientos se extienden de manera casi continua por más de 1,200 km, desde la frontera MéxicoEstados Unidos hasta la Faja Volcánica Transmexicana (FVTM), cubriendo una superficie de $-300,000 \mathrm{~km}^{2}$.
Durante el Mioceno, antes de la apertura del Golfo de California y del inicio de la actividad del FVTM, que la disgregaron y sepultaron parcialmente, la provincia magmática de la SMO debió ser considerablemente más extensa y estar unida a la provincia volcánica de Baja California (Figura 2). Las regiones que bordean al núcleo actual (sin una deformación por extensión muy manifiesta) de la SMO (Figura 3) son o fueron parte de la provincia tectónica de Cuencas y Sierras (CyS). un área vasta de la Cordillera Norteamericana que ha experimentado tectónica distensiva en el Cenozoico medio y tardío. En algunos sitios la extensión comenzó a partir del Paleógeno. Actualmente CyS abarca en 


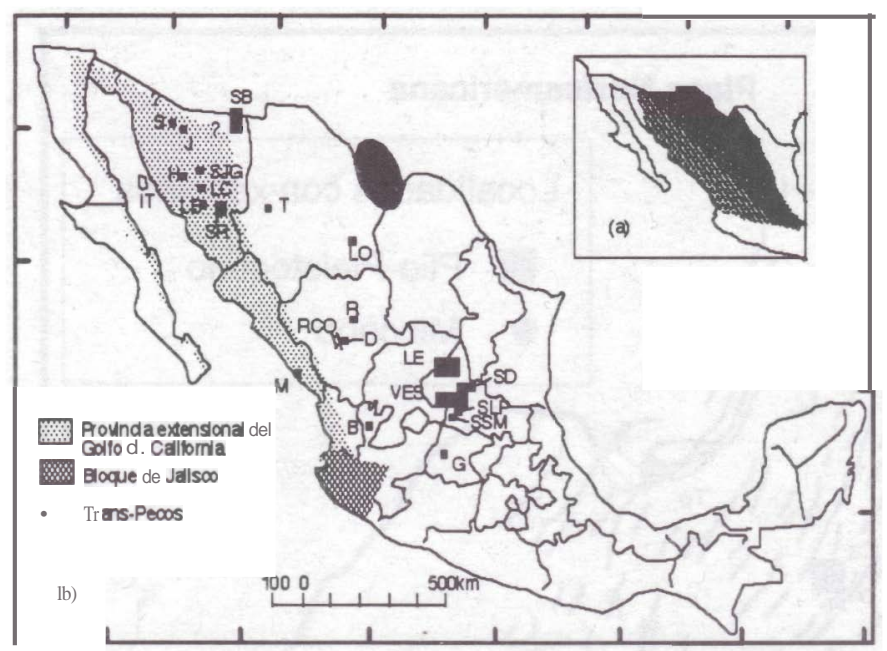

Figura 1. a. Mapa índice que muestra aproximadamente la región a la que se refiere esta revisión. b. Límites aproximados de provincias geológicas y localidades mencionadas en el texto. El tamaño y forma de los rectángulos negros es aproximadamente proporcional al tamaño de las áreas citadas. Las ciudades se representan como cuadros negros. Clave: $\mathrm{B}=$ Bolaños; $\mathrm{O}=$ Durango; $\mathrm{G}=$ Guanajuato; $\mathrm{H}=$ Hermosillo; IT =Isla Tiburón; $\mathrm{J}=$ Jarillas; $\mathrm{LB}=$ Lista Blanca; $\mathrm{LC}=$ La Colorada-Rancho San Francisco; LE = Los Encinos; LO = La Olivina; $\mathrm{M}=$ Mazatlán; $\mathrm{R}=$ Rodeo; $\mathrm{RCO}=$ Río Chico-Otinapa; $\mathrm{S}=$ Saric; $\mathrm{SB}=$ San Bernardino; $\mathrm{SO}=$ Santo Domingo; SJG = San José de Gracia; SLP = San Luis Potosí; SR = Santa Rosa; SSM = Sierra San Miguelito; T = Tomóchic; VES = Ventura-Espíritu Santo.

forma continua desde el SW de Canadá hasta el límite entre la Mesa Central y el FVTM, y antes de la formación de este arco volcánico pudo haberse prolongado hasta el sur de México (e.g., Henry y Aranda-Gómez, 1992; Jansma y Lang, 1997). Sin embargo, Morán-Zenteno et al. (éste volumen) hacen notar que al sur de la FVTM existen numerosas evidencias de fallas de desplazamiento lateral con orientaciones que varían de NNE a NNW, lo que contrasta con el fa-llamiento dominantemente normal en CyS. Como sucede en la parte septentrional de CyS, la porción mexicana puede ser dividida en varios dominios estructurales, que en detalle difieren en historia geológica y en grado y estilo de deformación, pero que en conjunto representan cambios en una serie continua.

La investigación sistemática de la geología de la región comenzó en los años setenta y ha seguido hasta la fecha. Por la extensión de las áreas cubiertas y/o por la continuidad del esfuerzo realizado destacan los grupos de investigadores que' documentaron e interpretaron: 1 . La edad de la secuencia volcánica en Durango y Sinaloa (e.g., McDowel1 y Keizer, 1977; Swanson et al., 1978; McDowell, 1979; Swanson y McDowell, 1984; Henry y Fredrikson, 1987; AguirreDíaz y McDowell, 1991), 2. La composición química y petrogénesis de las rocas de la SMO (e.g. , Cameron et al.,1980), 3. La estratigrafía volcánica, trama

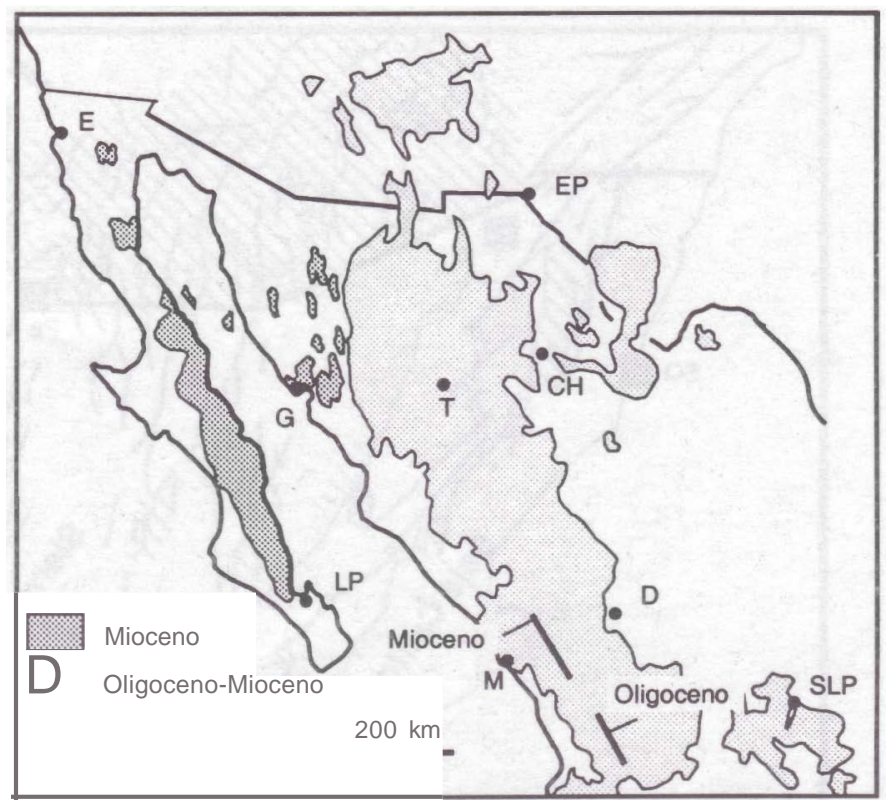

Figura 2. Mapa generalizado que muestra la distribución de rocas volcánicas asociadas a subducción del Oligoceno y Mioceno temprano en el NW de México. En la porción oriental de la cuenca del Golfo de California predominan las rocas volcánicas del Mioceno. Este contacto no ha sido cartografiado a lo largo de toda la SMO. Modificado de McDowell y Keizer (1977) y de Ortega-Gutiérrez y colaboradores (1992). Ciudades: $\mathrm{CH}=$ Chihuahua, $\mathrm{O}=$ Durango, $\mathrm{E}$ $=$ Ensenada, $\mathrm{EP}=\mathrm{El}$ Paso, GU = Guaymas, $\mathrm{LP}=\mathrm{La} \mathrm{Paz}, \mathrm{M}=$ Mazatlán, SLP $=$ San Luis Potosí.

estructural, y evolución geológica de la Mesa Central y del extremo meridional de la SMO (e.g., LabartheHernández et al.,1982; Tristán-González, 1986; ArandaGómez et al., 1989; Nieto-Samaniego et al., 1997, 1999), 4. Los patrones de inclinación de las rocas cenozoicas (Stewart y Roldán-Quintana, 1994; Stewart et al., 1998), 5. La petrogénesis de basaltos (sensu lato) alcalinos con xenolitos del manto (e.g., Aranda-Gómez et al., 1992; Luhr et al., 1989; 1995, 1998; Pier et al., 1989,1992).

A los trabajos iniciales de inventario y caracterización han seguido síntesis en que se analizan las relaciones entre magmatismo, tectónica, naturaleza del basamento continental y manto litosférico, y la dinámica de placas. Actualmente se están proponiendo modelos geodinámicos para el origen y evolución de la SMO y del fallamiento CyS, y la posible interrelación entre ambos fenómenos (e.g., Nimz et al., 1986, 1993, 1995; Ruíz et al., 1988; Smith et al., 1996; ArandaGómez et al., 1997; Henry y Aranda-Gómez, 1998; Nieto et al., 1999).

\section{EL CAMPO VOLCÁNICO DE LA SIERRA MADRE OCCIDENTAL}

La SMO generalmente es considerada producto del paroxismo ignimbrítico (ignimbrite flareup). Este 


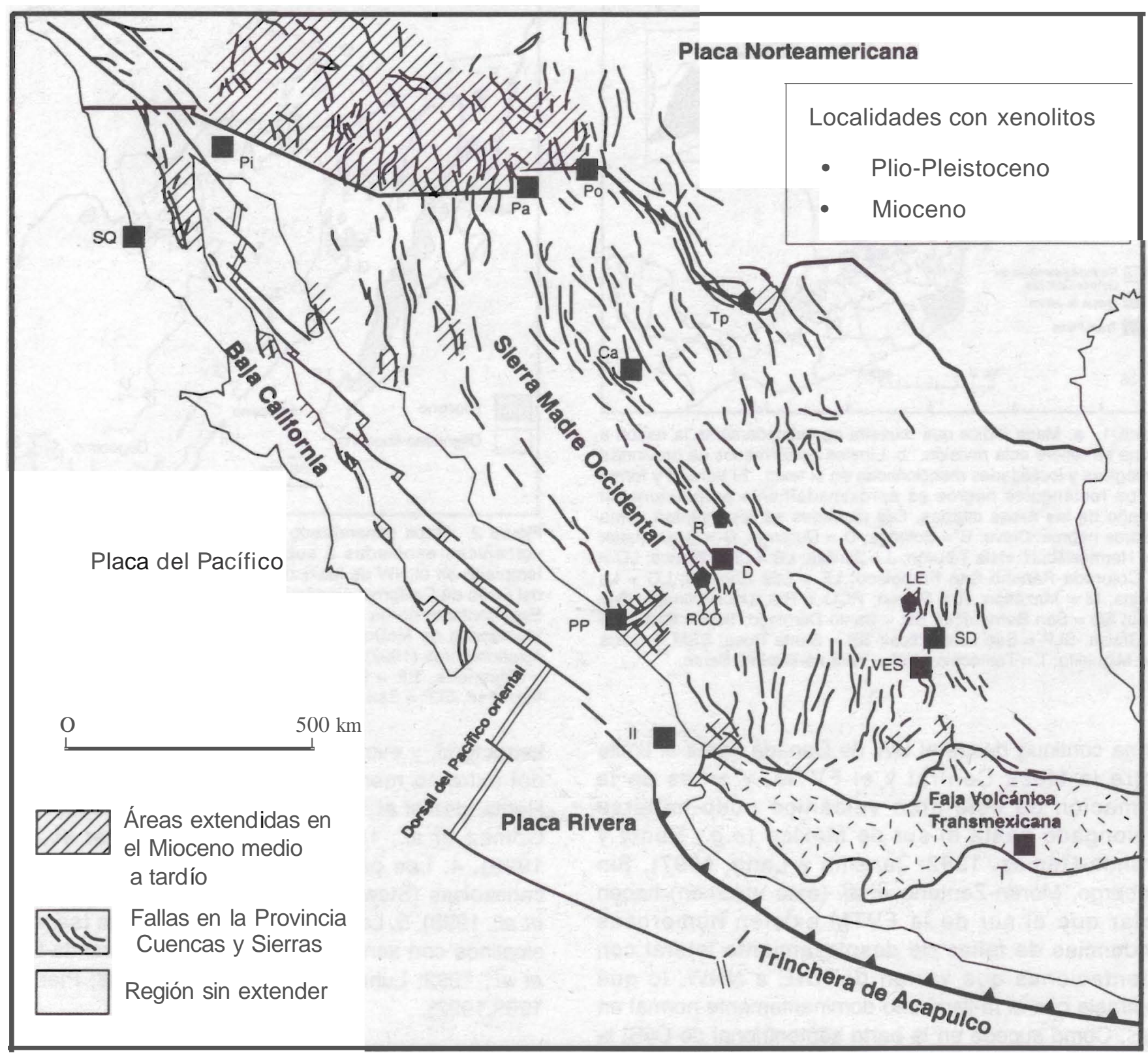

Figura 3. Extremo meridional de la provincia tectónica de Cuencas y Sierras. En el mapa también se muestran las localidades en donde se ha documentado extensión contemporánea al protogolfo de California durante el Mioceno medio a tardío. Las localidades con xenolitos del manto se encuentran en campos volcánicos con edades de 4.7 a $0.04 \mathrm{Ma}$. En algunas de las localidades con xenolitos el volcanismo fue contemporáneo a fallamiento normal (e.g., Camargo y Ourango). También se ha documentado fallamiento cuaternario en la porción norte de Chihuahua, especialmente en la cuenca del Río Bravo, entre Ciudad Juárez $(\mathrm{J})$ y Ojinaga $(\mathrm{O})$. Clave: Pi =Pinacate; $\mathrm{Pa}=\mathrm{Palomas;} \mathrm{Po}=\mathrm{Potrillo}$; Tp:Transpecos; $\mathrm{SQ}=$ San Quintín; $\mathrm{Ca}=$ Camargo; $\mathrm{R}=$ Rodeo; $\mathrm{O}=$ =Ourango; $\mathrm{M}$ =Metates; $\mathrm{PP}=$ =Punta Piaxtla; LE =Los Encinos; SO =Santo Domingo; VES =Ventura- Espíritu Santo; T =Temascaltepec, 11 = Isla Isabel.

evento sucedió en el lapso 32-28 Ma en el borde oriental del campo volcánico (Figuras 1 y 2: Chihuahua, Durango, Zacatecas, San Luis Potosí, Guanajuato y Jalisco), y se desplazó hacia la costa del Pacífico durante el Oligoceno tardío y el Mioceno temprano. Sin embargo, se ha señalado que abajo del Supergrupo Volcáníco Superior (K·Ar < 32 Ma: McDowell y Keizer, $19 n$ ) hay una secuencia volcanoplutónica del Eoceno que indica la existencia de un vasto campo volcánico en esa época ( guirre-Díaz y McDowell, 1991). Afloramientos aislados de capas rojas, parcialmente contemporáneos al volcanismo del Eoceno (Figura 4), y posiblemente acumuladas en fosas tectónicas activas durante el depósito, sugieren que hubo extensión cortical contemporánea a este campo volcánico predecesor de la SMO (Aranda-Gómez y McDowell, 1998).

\section{Origen de los magmas de la SMO}

Una de las controversias acerca de la provincia volcánica de la SMO se centra en la fuente de los 


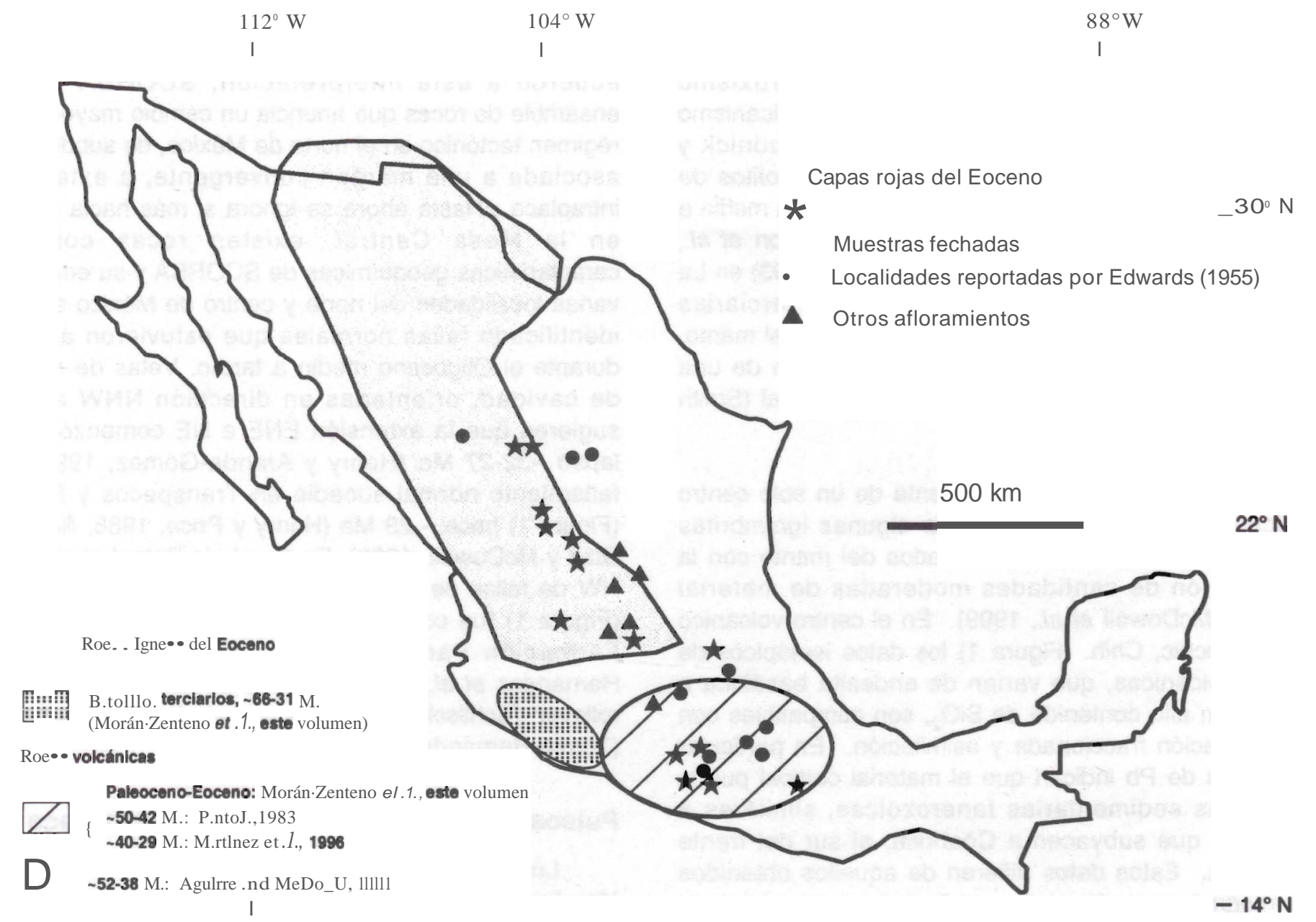

Figura 4. Extensión probable del arco volcánico del Eoceno. Al sur de la Faja Volcánica Transmexicana, en la región de Taxco, Gro., se han reportado rocas volcánicas del Eoceno al Oligoceno temprano. Se desconoce si hubo continuidad entre estos dos campos volcánicos. En la figura también se muestra los afloramientos de capas rojas; al menos en Guanajuato, y posiblemente zacatecas y Taxco, estas secuencias se asociaron a fallamiento normal.

magmas que dieron origen al Supergrupo Volcánico Superior, compuesto por una suite basalto-andesitariolita. I.a composición silícica dominante de la suite, su espesor medio $(-1 \mathrm{~km})$, y contenido isotópico (Nd-Sr), similar al de algunos de los xenol itos corticales del centro y norte de México, hacen atractivo considerar a estos magmas como productos de fusión parcial de la corteza, posiblemente causada por emplazamiento de un volumen similar de magmas basálticos provenientes del manto (Ruiz et al., 1988). Por otro lado, la composición química, elemental e isotópica, de las rocas volcánicas terciarias también puede modelarse por procesos de cristalización fraccionada de magmas basálticos del manto y asimilación simultánea de cantidades variables, aunque moderadas, de material cortical (Cameron y Robinson, 1990). Esta segunda opción requiere de adición de al menos de $4 \mathrm{~km}$ de agregados cumulofíricos de minerales máficos y plagioclasa a la corteza, haciendo de este pulso magmático un período importante de crecimiento cortical (Ruiz et al., 1990). Para resolver esta disyuntiva no basta conocer la composición isotópica de las rocas volcánicas y de los xenolitos corticales, sino que también se necesita saber la edad de ambos (Cameron y Robinson, 1990). Edades isotópicas (U-Pb: 25-37 y 20O Ma) obtenidas por Rudnick y Cameron (1991) en zircones de xenolitos de granulitas feldespáticas de La Olivina, Chih., indican que del Eoceno al Oligoceno medio hubo un pulso térmico que coincidió con el volcanismo de la SMO en el área, y que del Mioceno medio a la actualidad, en la parte media y profunda de la corteza existen temperaturas consistentes con metamorfismo de grado alto (í.e., facies de granulita). Condiciones similares de P-T también existen en la corteza de San Luis Potosí (Hayob et al.,1989) y, en general, en el centro de México, como infiere Fix (1975) de un estudio sismológico de dispersión de ondas superficiales que sugiere la presencia de una zona de 
baja velocidad y alta temperatura en esa región. Es posible que esto sea, al menos en parte, el vestigio de un pulso térmico mayor asociado al paroxismo ignimbrítico de la SMO o que se deban al volcanismo alcalino asociado a extension cortical (Audnick y Cameron, 1991). Estudios detallados de xenolitos de piroxenitas del manto y gra-nulitas de la corteza media e inferior (Nimz et al., 1986,1993, 1995; Cameron et al., 1992), y de ignimbritas oligocénicas (Smith, 1993) en La Olivina (Figura 1) indican que las rocas terciarias evolucionaron a partir de magmas basálticos del manto, por cristalización fraccionada con asimilación de una cantidad modesta « 20\%) de paragneis cortical (Smith et al., 1996).

Un estudio exhaustivo reciente de un solo centro volcánico en la SMO indica que algunas ignimbritas evolucionaron de basaltos derivados del manto con la asimilación de cantidades moderadas de material cortical (McOowell et al., 1999). En el centro volcánico de Tomóchic, Chih. (Figura 1) los datos isotópicos de rocas volcánicas, que varían de andesita basáltica a riolita con alto contenido de $\mathrm{SiO}_{2}$, son compatibles con crista-lización fraccionada y asimilación. En particular los datos de $\mathrm{Pb}$ indican que el material cortical puede ser rocas sedimentarias fanerozoicas, similares a aquellas que subyacen a Coahuila, al sur del frente Ouachita. Estos datos difieren de aquellos obtenidos de magmas portadores de xenolitos del manto del norte de México, lo que parece excluir su derivación directa del manto, sin interacción con la corteza.

\section{Inicio de la extensión y cambios de compo- sición en los magmas asociados a subducción}

Entre 29-20 Ma fueron extravasadas en Chihuahua, sur de Sonora y norte de Sinaloa lavas que en conjunto tienen composiciones distintas a las de la suite asociada a subducción basalto-andesita-riolita de la SMO y las de los basaltos (sensu iato) intraplaca del Oligoceno tardío-Cuaternario. Cameron et al. (1989) llamaron a este conjunto de rocas Southem Cordillera Basaltic Andesite (SCOABA) e indicaron que esta puede ser la provincia basáltica más extensa del Cenozoico de Norteamérica, ya que existen rocas similares en una región muy extensa del suroeste de los Estados Unidos. SCOABA esta formada principalmente por andesitas basálticas asociadas a subducción. En comparación con la suite basalto-andesita-riolita de la SMO, SCOABA tiene un contenido promedio menor de $\mathrm{SiO}_{2}$. Se cree que SCOABA fue extravasada a través de una corteza más extendida que la que existía previamente,! que estos magmas más primitivos llegaron más rápidamente a la superficie, con menos diferenciación que los magmas claramente asociados a una margen convergente (Cameron et al., 1989). De acuerdo a esta interpretación, SCOABA es un ensamble de rocas que anuncia un cambio mayor en el régimen tectónico en el norte de México, de subducción asociada a una margen convergente, a extensión intraplaca. Hasta ahora se ignora si más hacia el sur, en la Mesa Central, existen rocas con las características geoquímicas de SCOABA y su edad. En varias localidades del norte y centro de México se han identificado fallas normales que estuvieron activas durante el Oligoceno medio a tardío. Vetas de relleno de cavidad, orientadas en dirección NNW a NW, sugieren que la extensión ENE a NE comenzó en el lapso -32-27 Ma (Henry y Aranda-Gómez, 1992). El fallamiento normal sucedió en Transpecos y Aodeo (Figura 1) hace - 29 Ma (Henry y Price, 1986; AguirreOíaz y McOowell, 1993). En San Luis Potosí el sistema NW de fallas de dominó de la sierra de San Miguelito (Figura 1) fue contemporáneo a la acumulación de la Formación Panalillo (K-Ar -26.8 Ma; LabartheHernández et al., 1982) como lo demuestra un pliegue rollover (Schlische, 1995) documentado en esta unidad (Torres-Hernández, 1998).

\section{Pulsos de extensión y volcanismo intraplaca}

Los magmas intraplaca más antiguos (K-Ar: 24-20 Ma; Aguirre-Oíaz y McOowell, 1993) conocidos en la región son las hawaiitas de Aodeo, Ogo. (Figura 1). Estas rocas son similares en edad y composición a lavas de la región de Transpecos en Texas (Luhr et al., 1998). Las hawaiitas de Aodeo hicieron erupción cerca de la falla maestra (breakaway 'ault zone) de un semigraben mayor (Aranda-Gómez et al., 1997) y se encuentran intercaladas con depósitos clásticos de relleno de graben (i.e., las Gravas Santa Inés), demostrándose así la contemporaneidad del magmatismo alcalino con un pulso importante de extensión cortical.

En el siguiente pulso, el Basalto Metates (K-Ar -12 Ma; McOowell y Keizer, 1977) fue extravasado un poco después del inicio de la extensión en el Graben del Aío Chico, Ogo. (Figura 1). La composición y edad del Basalto Metates son muy similares a la de las hawaiitas del campo volcánico de Los Encinos (SLPZac: Figura 1). A diferencia de las rocas alcalinas máficas del Plio-Cuaternario, las hawaiitas de Los Encinos y Metates carecen de xenolitos del manto, conteniendo en cambio conjuntos complejos de megacristalesaccidentales (feldespato, kaersutita, olivino, magnetita, apatito, espinela), agregados policristalinos esporádicos con mineralogía semejante 
a la del conjunto de megacristales y algunos xenolitos de granulitas feldespáticas semidigeridas. Las hawaiitas miocénicas (K-Ar-13-10 Ma) de Los Encinos muestran evidencias tísicas (xenocristales de cuarzo abundantes y sus productos de reacción con la lava anfitrión) y químicas (elemental e isotópicas) de contaminación intensa con material cortical. Por tanto, se cree que ascendieron más lentamente que los magmas alcalinos cuaternarios $(\mathrm{K}-\mathrm{Ar}<1.4 \mathrm{Ma}$; Aranda-Gómez y Luhr, 1996) que ori-ginaron los campos volcánicos de Santo Domingo y VenturaEspíritu Santo (Figura 1), ubicados inmediatamente al sur de Los Encinos. El ascenso relativamente lento de los magmas miocénicos les permitió perder sus xenolitos del manto, diferenciarse por cristalización fraccionada y fundir y asimilar material cortical. Algunos.de los megacristales al parecer son derivados de magmas similares a las hawaiitas del Mioceno, aunque ligeramente más diferenciados. Se cree que algunos de los magmas no lograron llegar a la superficie, cristalizaron lentamente a gran profundidad. Posteriormente, estos cuerpos plutónicos fueron desagregados al paso de magmas más jóvenes, quienes transportaron los megacristales y xenolitos a la superficie (Luhr et al., 1995).

\section{Origen de la extensión en la porción meri- dional de Cys}

Se han propuesto muchas hipótesis acerca del origen de CyS. Sonder y Jones (1999) recientemente publicaron una revisión crítica acerca de los modelos propuestos para el oeste de los Estados Unidos, excluyendo a la porción mexicana de CyS. Las hipótesis pueden agruparse en varios tipos extremos como:

1. Las que apelan a procesos interplacas (j.e., debidos a fuerzas diversas generadas por las interacciones entre la placa Norteamericana con las placas Farallón y/o Pacífica, e.g., Severinghaus y Atwater, 1990),

2. Aquellas que asocian la extensión a procesos subcorticales (e.g., levantamiento o formación de una "ampolla" en el manto (e.g., Scholz et al., 1971).

3. Las que emplean mecanismos intraplaca, principalmente la energía potencial gravitacional generada por engrosamiento local de la corteza por plegamiento y/o magmatismo (e.g., Wernicke et al., 1987). Dado que ninguno de los tipos extremos es capaz por si solo de satisfacer la complejidad de los fenómenos involucrados en la formación de la porción de CyS en el oeste de los Estados Unidos, también se han propuesto modelos que combinan dos o más de estos mecanismos extremos (e.g., Sonder y Jones, op. cit.).
En comparación con el oeste de los Estados Unidos, la cantidad de información geológica y geofísica disponible acerca de la porción mexicana de CyS es muy limitada. Consecuentemente, el número de modelos también es más reducido $\mathrm{y}$, como se verá mas adelante, sólo aspiran a explicar fenómenos aislados.

La extensión alrededor del Golfo de California (i.e., la Provincia Extensional del Golfo de California (Figura 1) es interpretada como el resultado del movimiento oblicuo entre las placas Pacifica y Norteamericana. El desplazamiento inicial puede descomponerse en un vector extensional, perpendicular al márgen continental, y en un vector de desplazamiento a rumbo, paralelo a este, a lo largo de la falla Tosco-Abreojos (Stock y Hodges, 1989) o como producto de transtensión desde su comienzo (Gans, 1997). La formación del Protogolfo (-12-10 Ma) coincide con un período de extensión y volcanismo alcalino al E de la SMO, y con fallamiento normal en las áreas ubicadas inmediatamente al norte y sur del núcleo sin extender de la SMO (Figura 3). Por tanto, el pulso de extensión y volcanismo alcalino en la región posiblemente obedece a la misma causa (Henry y Aranda-Gómez, 1999).

La cronología, intensidad y dirección de los pulsos de extensión terciaria han sido analizados en varias regiones de CyS. Aunque existen eventos comunes, que se repiten a través de la provincia, en detalle la historia puede diferir de un área a otra. Por ejemplo, en Sonora, existen indicios de que en la Isla Tiburón (Figura 1) la extensión ya había comenzado hace $30 \mathrm{Ma}$ (Calmus, 1998). La edad (40Ar- ${ }^{39} \mathrm{Ar}$ : $\left.28 \mathrm{Ma}\right)$ del core complex de Jarillas (Figura 1) indica que hubo hiperextensión en esa región (Herrera-Urbina et al., 1995). En la región de Santa Rosa, Son., hubo extensión temprana (-N500W) de gran magnitud (90\%) que produjo fallas, con rumbo NW y ángulo de inclinación de mode-rado a bajo, en el lapso 27-20 Ma (Gans, 1997). Las secuencias afectadas fueron basculadas hasta $60^{\circ}$. Después, entre 20 y $17 \mathrm{Ma}$, hubo extensión E-W «10-15\%), que solo causó basculamientos modestos. Los basaltos y andesitas postectónicos « $17 \mathrm{Ma}$ ) permanecen subhorizontales en la región de Santa Rosa (Gans, 1997). Fallas normales de ángulo alto cortan rocas volcánicas de 12-10 Ma (Bartolini et al., 1993) en las regiones de San José de Gracia, Sierra Lista Blanca y La Colorada-Rancho San Francisco (Figura 1). Al igual que en la fase tectónica anterior, este pulso solo causó basculamientos pequeños $\left(4-11^{\circ}\right)$. El fallamiento histórico (1 887) en el valle de San Bernardino (Figura 1), al noreste del estado, causó ruptura a lo largo de $75 \mathrm{~km}$ en la falla de 
Pitaycachi (Natali y Sbar, 1982).

En Durango también se ha reconocido fallamiento multiepisódico. La primer etapa de fallamiento en Rodeo sucedió -29 Ma. Un segundo pulso (-24-20 Ma) produjo basculamientos hasta de $35^{\circ}$ en Rodeo. El graben del Río Chico se formó en el lapso 12-13 Ma y causó inclinaciones de hasta $12^{\circ}$. El último pulso «2.5 Ma: Aranda-Gómez y Henry, 1992) desplazó e inclinó casi imperceptiblemente a basaltos alcalinos plio-cuaternarios en el campo volcánico de Durango (Figura 1). Microsismicidad histórica (Yamamoto, 1993) indica que la extensión continúa activa en el área.

\section{Extensión en la Mesa Central}

El papel que jugaron las estructuras pre-existentes en la reactivación simultánea de sistemas ortogonales (NE y NW) de fallas normales cenozoicas en la Mesa Central fue analizado por Nieto et al. (1997), concluyéndose que durante el Cenozoico medio y tardío la deformación fue triaxial, con alargamiento principal máximo $(-20 \%)$ aproximadamente E-W. Los sistemas de fallas reactivados posiblemente fueron generados en varios pulsos de extensión durante el Paleógeno (Aranda-Gómez y McDowell, 1998).

\section{Migración del volcanismo y fallamiento}

Durante el Oligoceno y Mioceno la migración del frente volcánico hacia la trinchera está bien documentada en la porción septentrional de la SMO (Clark et aL, 1982). En Sonora la mayor parte de la extensión CyS sucedió entre 30 y 17 Ma, simultáneamente a la subducción de vestigios de la placa Farallón y a la migración hacia el Oeste del arco volcánico. La extensión fue acompañada por volcanismo máfico en las fases iniciales (Gans, 1997). La edad mínima de la Formación Baucarit (-10 Ma: McDowell y Roldán-Quintana, 1993; McDowell et aL, 1997), un depósito clástico continental que se cree acumulado en cuencas asociadas a extensión, y que en lugares está intercalado con rocas volcánicas, sugiere que la deformación y volcanismo asociado a subducción pudo continuar hasta el Mioceno temprano (Stewart y Roldán-Quintana, 1994). Magmatismo intraplaca, basaltos alcalinos y hawaiitas (-7 Ma: PazMoreno, 1998), posiblemente asociado a extensión, sucedió en la región de Saric (Figura 1).

En el extremo meridional de la SMO y de CyS se ha documentado una migración similar de la actividad magmática asociada a subducción que fue acompañada, o.seguida después de un lapso breve, por pulsos de extensión importantes en las mismas regiones donde sucedió el volcanismo. Las etapas paroxísmicas de magmatismo asociado a subducción y extensión (29-27 Ma cerca de la ciudad de San Luis Potosí, 23-19 Ma en la región de Bolaños; véase Figura 1) coinciden con períodos en que se incrementó notablemente la tasa de divergencia entre las placas Farallón y Pacífica, al sur de la zona de fractura Shirley, posiblemente debido a tasas más rápidas de subducción, que a su vez indujeron magmatismo y fallamiento extensional en la placa continental por efecto de un retroceso de la trinchera (Nieto-Samaniego et al., 1999). Hacia el noreste, simultáneo a los pulsos de volcanismo asociado a subducción, hubo expulsión de basaltos intraplaca.

En general, toda la provincia CyS, desde la frontera EUA-Canadá hasta la FVTM (Figura 5), muestra esta migración de la extensión hacia el $\mathrm{W}$ o SW (Stewart, 1998). En el noroeste de México volcanismo y extensión fueron contemporáneos, o el fallamiento sucedió un poco después del volcanismo.

\section{SUMARIO DE LA EVOLUCIÓN TECrONO- MAGMÁTICA DE LA REGIÓN}

En las Figuras 6 y 7 se resume la información presentada en este trabajo acerca de la evolución tectonomagmática del extremo meridional de CyS. Esto se hace de manera esquemática, por medio de diagramas de barra, similares a los diagramas paragenéticos. La Figura 6 correponde a localidades situadas al este de la SMO, desde el Río Grande hasta el límite septentrional de la FVTM. La Figura 7 representa una sección transversal (E-W) de la provincia, entre Durango y Mazatlán. Algunos de los rasgos más sobresalientes de los diagramas son: 1. En los períodos 30-27,24-20, 13-10 Y $<5$ Ma hubo actividad magmática y pulsos de extensión simultáneos, - casi simultáneos a través de toda la porción meridional de CyS; 2. La composición y volumen de las lavas en cada uno de estos períodos varió de un lugar a otro. Así, durante el pulso del Mioceno temprano (24$20 \mathrm{Ma}$ ) en Chihuahua y el oeste de Texas eran extravasados magmas intraplaca sin xenolitos del manto, mientras que Guanajuato y el núcleo de la Sierra Madre Occidental (Figura 1) sucedía volcanismo calcialcalino asociado a subducción. En el mismo lapso la actividad volcánica calcialcalina llegaba a su fin en Guanajuato (Figura 1), mientras que en el núcleo de la SMO se formaba la voluminosa secuencia del Espinazo-El Salto (McDowell y Keizer, 1977). De manera similar, en el lapso 13-10 Ma, en Durango, San Luis Potosí y Zacatecaseran expulsados magmas intraplaca, mientras que en Sinaloa y Sonora (e.g., 


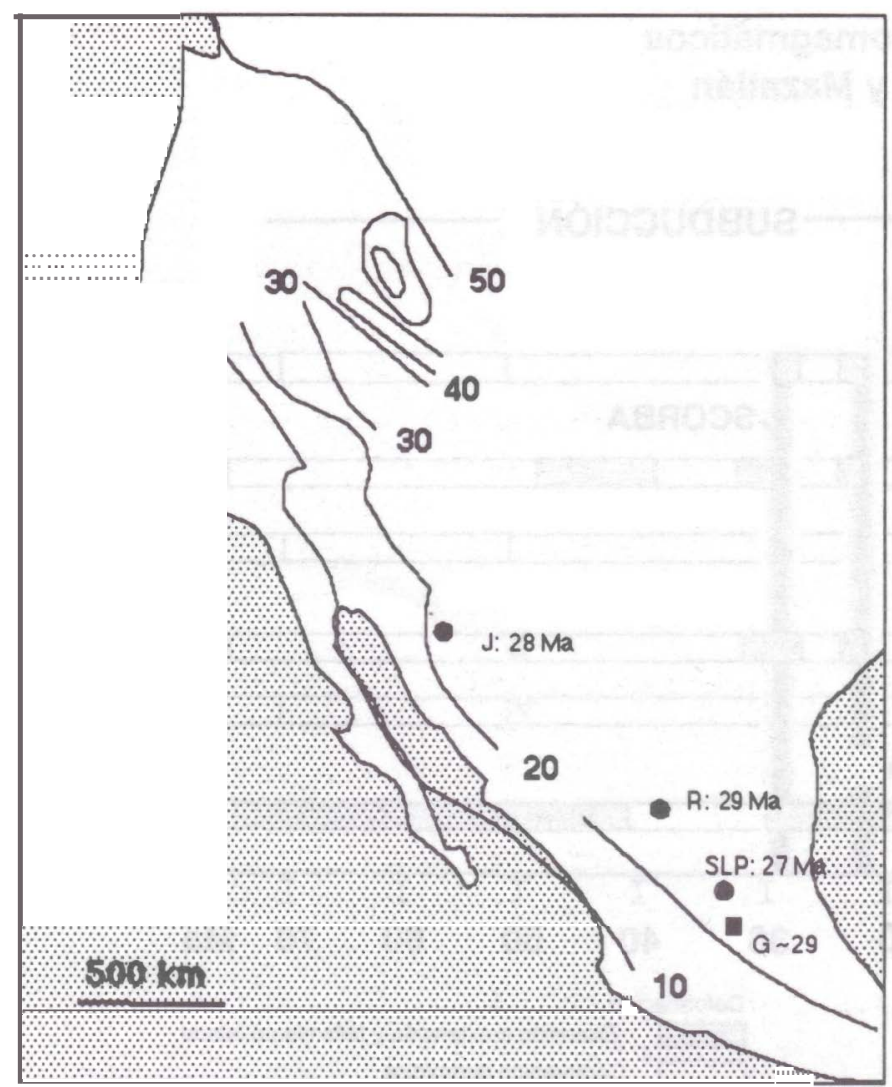

Mora-Álvarez y McDowell, en prensa; Henry y ArandaGómez, 1998) se emplazaron diques y derrames
Figura 5. Curvas de Isovalores correspondientes a edades generalizadas de fallas mayores de ángulo bajo (detachment faults) o basculamientos mayores de $25^{\circ}$ en rocas estratificadas (Stewart, 1998). En Rodeo (R), San Luis Potosí (SLP) y Guanajuato (G) hubo extensión en los tiempos señalados. Solo en Rodeo existió basculamiento mayor de $25^{\circ}$. La extensión comenzó en Guanajuato durante el Eoceno. El core complex de Jarillas $(\mathrm{J})$ tiene una edad de 3OMa.

toléticos. 3. El cambio de volcanismo asociado a una margen convergente a magmatismo intraplaca varió sistemáticamente en edad de norte a sur y de este a oeste. Al parecer la componente de desplazamiento $\mathrm{N}$ $S$ fue menor, y menos evidente, que la E-W. La resultante de estas componente es un desplazamiento neto de NE a SW.

\section{TRANSICIÓN SMO-FVTM y OTROS EFECTOS DE LA REORGANIZACIÓN DE PLACAS}

Durante el Oligoceno el arco magmático de la SMO tuvo una orientación NNW a NW, subparalelo a la costa del Pacífico, y la composición dominante de sus rocas fue silícica. A partir del Mioceno medio el volcanismo asociado a subducción se concentró a lo largo de una franja E-W (i.e., la FVTM), que cruza al continente, y su composición fue principalmente andesítica a basáltica. Ferrari y colaboradores (1999)

Cambios tectonomagmáticos al este de la Sierra Madre Occidental

Protogolfo

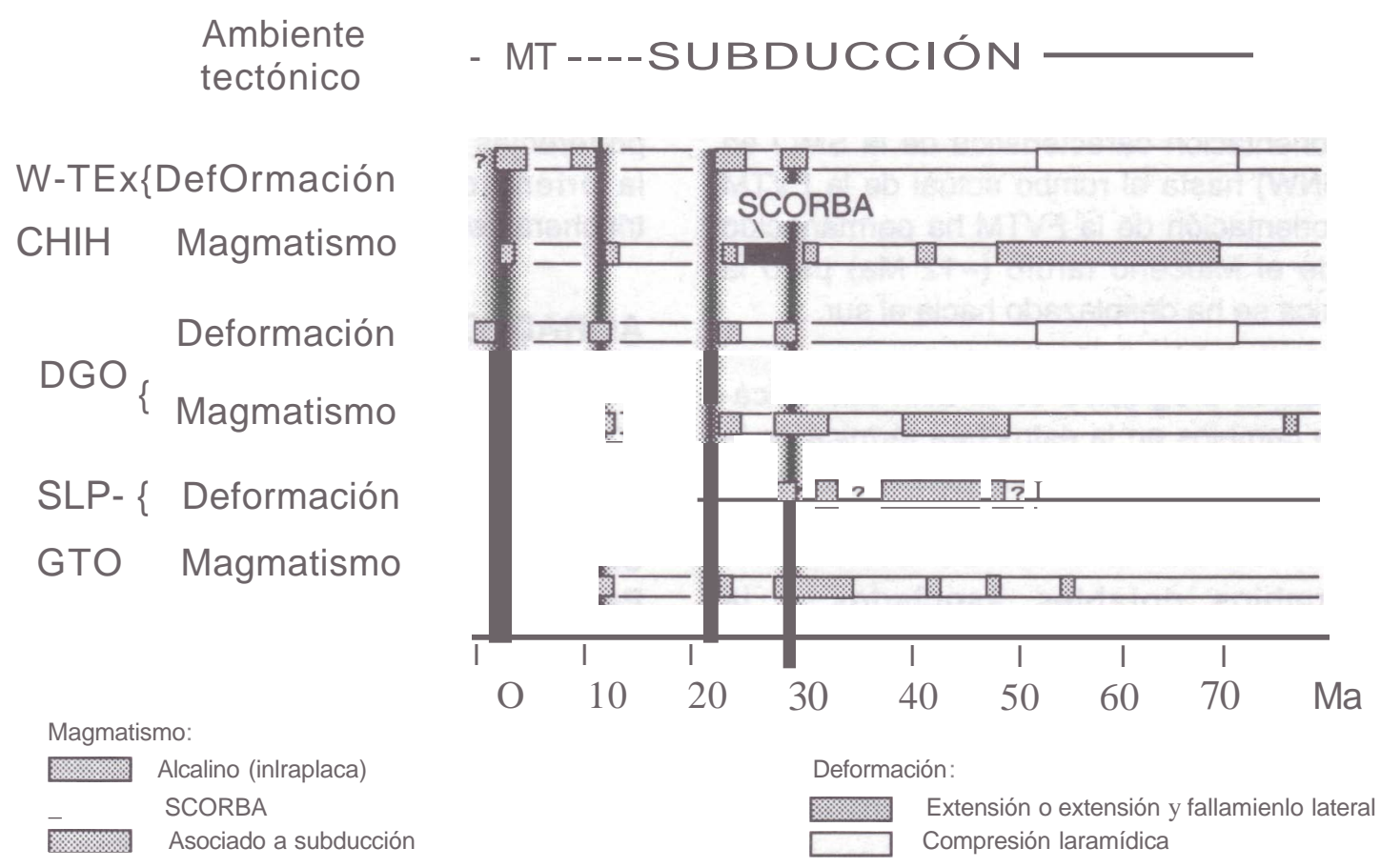

Figura 6. Resumen de los principales cambios tectonomagmáticos al este de la SMO. Los principales cambios en el ambiente tectónico son referidos a la región del Golfo de California: la apertura del protogolfo aproximadamente marca el fin de la subducción y el inicio de la actividad de tipo márgen transformante (MT). 


\section{Cambios tectonomagmáticos entre Durango y Mazatlán \\ Protogolfo}

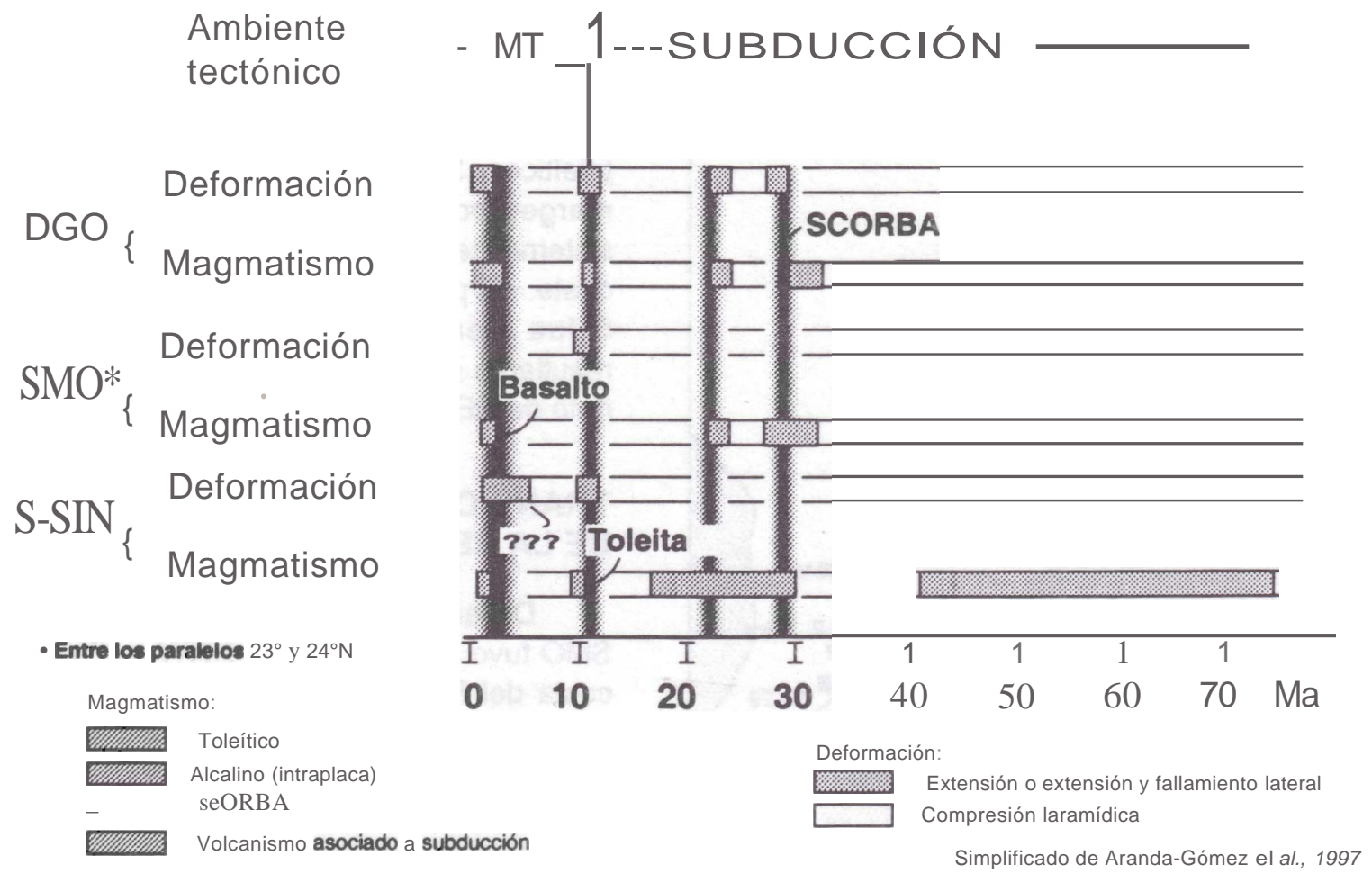

Figura 7. Variaciones en la edad y composición de las rocas volcánicas entre Durango y Mazatlán. También se muestran los pulsos de extensión reportados. SMO se refiere al núcleo casi sin extender, entre los paralelos $23^{\circ}$ y $24^{\circ} \mathrm{N}$

en un análisis estadrstico de las variaciones espaciotemporales del volcanismo en el centro de México muestran que la orientación del frente volcánico fue girando en sentido contrario al de las manecillas del reloj, desde la orientación característica de la SMO en el Oligoceno (NNW) hasta el rumbo actual de la FVTM (Figura 8). La orientación de la FVTM ha permanecido constante desde el Mioceno tardío (-12 Ma) pero la actividad volcánica se ha desplazado hacia el sur.

La reorientación progresiva de la actividad volcánica se asocia a cambios en la estructura térmica de la placa consumida y a variaciones en la geometría de los Irmites entre las placas (Ferrari et al., 1999).

Otros cambios notables asociados a la reorganización de las placas son que las rocas de la porción meridional de la SMO fueron plegadas en el Mioceno medio por transpresión lateral-izquierda a lo largo del límite con el Bloque de Jalisco (Figura 1) Y la $\mathrm{SMO}$. Este fenómeno estuvo asociado a la apertura del Protogolfo (Ferrari, 1995).

Asociado a la transición SMO-FVTM hubo deformación transcurrente, lo que parece indicar que el volcanismo inicial en la FVTM fue controlado por un sistema continental de fallas transtensionales izquierdas, quienes constituyeron conductos preferentes para el ascenso del magma y determinaron la orientación oblicua de la FVTM respecto a la trinchera de Acapulco (Ferrari et al., 1994).

\section{AGRADECIMIENTOS}

En los últimos 15 años nuestro trabajo en la porción mexicana de CyS ha sido financiado por varias agencias. Aranda-Gómez agradece el apoyo de CONACYT (proyectos: 2972-T9304 Y 3657PT) y de la UNAM (proyectos internos del Instituto de Geologra y PAPIIT IN114198). Henry recibió apoyo económico del Bureau of Economic Geology de la Universidad de Texas en Austin. El trabajo de Luhr fue financiado por National Science Foundation (EAR-8816987) y por el Smithsonian's Scholarly Studies Programo

A través del tiempo, nuestra colaboración y discusiones con muchos geólogos han influido en nuestras interpretaciones acerca de los temas 

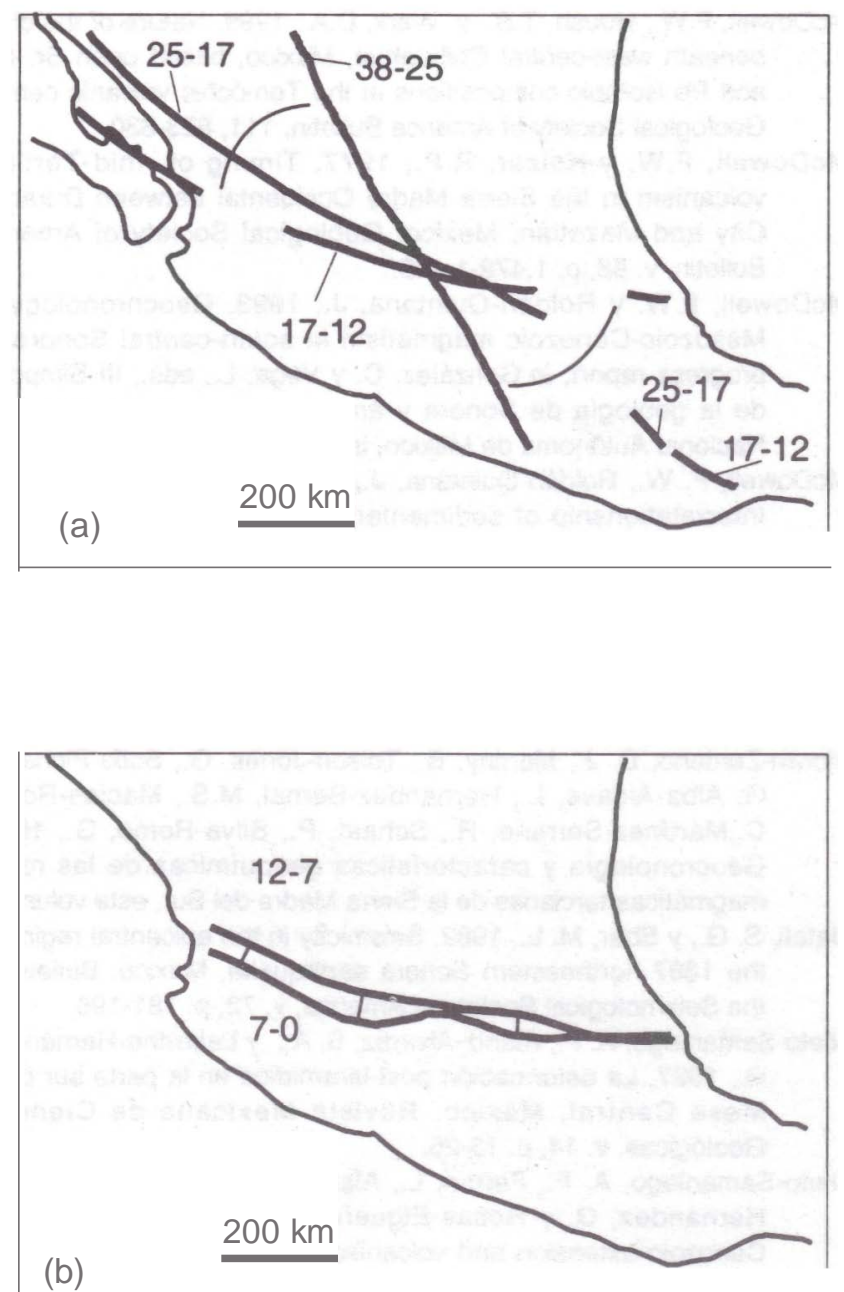

Figura 8. Variaciones espacio-temporales en la orientación de los arcos magmáticos cenozoicos. Cada línea fue trazada longitudinalmente en la porción media de áreas en las que se presentan rocas volcánicas de las edades especificadas. a. Durante el Oligoceno (38-25 Ma) el arco fue excepcionalmente ancho (-300 $\mathrm{km})$. En los otros períodos el ancho promedio fue menor (-150-200 $\mathrm{km})$. En el Mioceno medio (17-12 Ma) el arco se fragmentó, hubn un cambio substancial en la composición de los magmas, y adquirió la orientación que mantiene hasta ahora el FVTM. b. A partir del Mioceno tardío el frente volcánico del FVTM se desplazó hacia el sur. Simplificado de Ferrari el al., 1999.

discutidos en esta revisión. Nos sentimos especialmente en deuda con Guillermo Labarthe, Fred McDowell, Luca Ferrari, Todd Housh y Jean Pier. La revisión critica y comentarios hechos por Dante Morán, Luca Ferrari y Susana Alaniz ayudaron a mejorar este artrculo.

\section{REFERENCIAS BIBLIOGRÁFICAS}

Aguirre-Díaz, G. J., Y McDowell, F. W., 1991, The volcanic section at Nazas, Durango, Mexico, and the possibility of widespread Eocene volcanism within the Sierra Madre OCcidental: Joumal of Geophysical Research, v. 96, p. 13,373-13,388.

Aguirre-Díaz, G. J., Y McDowell, F. W., 1993, Nature and timing of faulting and synextensional magmatism in the southem Basin and Range, central-eastern Durango, Mexico: Geological Society of America Bulletin, v. 105, p. 1,435-1,444.
Aranda-Gómez, J.J., Aranda-Gómez, J.M., y Nieto-5amaniego, A.F. 1989, Consideraciones acerca de la evolución tectónica durante el Cenozoico de la Sierra de Guanajuato y la parte meridional de la Meseta Central: Universidad Nacional Autónoma México, Instituto de Geología, Revista, v. 8, n. 1, p.33-46.

Aranda-Gómez, J.J. y Henry, C.D., 1992, Fallamiento cuatemario cerca de la ciudad de Durango--naturaleza del período de deformación más joven relacionado a extensión multiepisódica en el noroeste de México: GEOS, v. 12, n. 5, p.53-54.

Aranda-Gómez, J. J., Henry, C. D., Luhr, J. F. Y McDowell, F. W., 1997, Cenozoic volcanism and tectonics in NW Mexioo a transect across the Sierra Madre OCcidental volcanic fieId and observations on extension related magmatism in the southem Basin and Range and Gulf of Califomia tectonic provinces, in G. J. Aguirre-Díaz, J. J. Aranda-G6mez, G. Carrasco-Nuñez, y L. Ferrari, eds., Magmatism and tectonics in the central and northwestem Mexico a selection of the 1997 IAVCEI General Assembly excursions: México, D.F., Universidad Nacional Autónoma de México, Instituto de Geología, p. 41-84.

Aranda-Gómez, J.J., y Luhr, J.F., 1996, Origin of the Joya Honda maar, San Luis Potosí, México: Joumal of Volcanology and Geothermal Researeh, v. 74, p. 1-18.

Aranda-Gómez, J. J., Luhr, J. F., Y Pier, J. G., 1992, The La Breña-S Jagüey maar complex, Durango, Mexico: 1. Geological evolution: Bulletin of Volcanology, v. 54, p. 393-404.

Aranda-Gómez, J. J., Y McDowell, F. W., 1998, Paleogene extension in the southem Basin and Ranga Province of Mexico-Syndepositional tilting of Eocene red bads and Oligocene volcanic rocks in the Guanajuato mining district:

Review, v. 40, p. 116-134.

Bartolini, C., Morales-Montaño, M. y Shafiqullah, M., 1993, Tertiary stratigraphy and geochronology (K-Ar) of the San José de Gracia area, central Sonora, Mexico: 111 Simposio de la geología de Sonora y áreas adyacentes, Universidad Nacional Autónoma México, Instituto de Geología, p. 13-15.

Calmus, T., 1998, Fechamiento por huellas de fisión: método y primeras aplicaciones en Sonora, México: Universidad Nacional Autónoma México, Instituto de Geología, Geología del Noroeste, 2 (2), 24-28.

Cameron , K.L., Cameron, M. , Bagby, W.C., Moll, E.J. Y Drake, RE., 1980, Petrologic characteristics of mid-Tertiary volcanic suites, Chihuahua, Mexico: Geology, v. 8, p.87-91.

Cameron, K. L., Nirnz, G. J. Y Kuentz, D., 1989, Southem CordUlera Basaltic Andesite Suite, Southem Chihuahua, Mexico-a link between Tertiary continental are and Ilood basalt magmatism in Northem America: Journal of Geophysical Research, v. 94, p. $7,817-7,840$.

Cameron, K. L., Y Robinson, J. V., 1990, Comments on "Nd-Sr isotopic compositions of lower crustal xenoliths-Evidence for the origin of mid-Tertiary volcanics in Mexico" by Ruiz, J., Patchett, P.J. y Arculus, R.J: Contributions lo Mineralogy and Petrology, v. 104, p. 609-614.

Cameron, K.L., Robinson, J.V., Niemeyer, S., Nirnz, G.J., Kuentz, D.C., Harmon, RS., Bohlen, S.R y Collerson, K.O., 1992, Contrasting styles of pre-Cenozoic and mid-Tertiary crustal evolution in northern Mexico-evidence from deep crustal xenoliths from La Olivina: Joumal of Geophysical Research, vol.97, p.17,353-17,376.

Clark, K. F., Foster, C. T. y Damon, P. E., 1982, Cenozoic mineral deposits and subduction-related magmatic ares in Mexioo: Geological Society of America Bulletin, v. 93, p. 533-544.

Edwards, J. D., 1955, Studies of some earty Tertiary red oonglomerates of central Mexico: US. Geological Survey, Professional Paper, vol. 264-H, p. 153-183.

Fix, J.E., 1975, The crust and upper mantle of central Mexico: Geophysical Journal, v. 43, n. 2, p. 455-497. 
Ferrari, I., 1995, Miocene shearing along the northem boundary of the Jalisco Block and the opening of the southem Gulf of Califomia: Geology, v. 23, p. 751-754.

Ferrari, I., Garduño, V. H., Pasquaré, G. y Tibaldi, A., 1994, Volcanic and tectonic evolution of central Mexico-Oligocene to present: Geofísica Intemacional, v. 33, p. 91-105.

Ferrari, I., lópez-Martínez, M., Aguirre-Díaz, G.J. y Carrasco-Nuñez, G., 1999, Space-time patterns of Cenozoic arc volcanism in central Mexico-from the Sierra Madre Occidental to the Mexican Volcanic Belt: Geology, v. 27, p. 303-306.

Gans, P. B., 1997, large-magnitude Oligo-Miocene extension in southern Sonora- Implications for the tectonic evolution of northwestern Mexico: Tectonics, v. 16, p. 388-408.

Hayob, J.I., Essene, E.J., Ruiz, J., Ortega-Gutiérrez, F. y ArandaGómez, J.J., 1989, Very high-temperature deep-crustal metamorphism revealed in pelitic xenoliths from central Mexico: Nature, v. 342, p. 265-268.

Henry, C. D., y Aranda-Gómez, J. J.: 1992, The real southern Basin and Range:mid-to late Cenozoic extension in Mexico: Geology, v. 20 , p. $701-704$.

Henry, C. D., y Aranda-Gómez, J. J., En prensa, Plate interactions control middle-late Miocene, proto-Gulf and Basin and Range extension in the southem Basin and Range: Tectonophysics.

Henry, C.D., y Fredrikson, G., 1987, Geology of part of southern Sinaloa, Mexico adjacent to the Gulf of Califomia: Boulder, CO, Geological Society of America, Map and Chart Series MCH063, 1 mapa

Henry, C.D., y Price, J.G., 1986, Earty Basin and Range development in Trans-Pecos Texas and adjacent Chihuahua-magmatism and orientation, timing, and style of extension: Journal of Geophysical Research, v. 91, núm.B6, p. 6,213-6,224.

Herrera-Urbina, S., Ortega-Rivera, M.A., Farrar, E. y Cochemé, J.J., 1995, Some apparent ${ }^{40} \mathrm{Ar} /{ }^{39} \mathrm{Ar}$ ages for metamorphic rocks in north central Sonora, Mexico, and their interpretation: Boletín del Departamento de Geología, Uni-Son (México), v. 12, n. 1, p. 85-100.

Jansma, P.E. y lang, H.R., 1997, The Arcelia graben-New evidence for Oligocene Basin and Range extension in southem Mexico: Geology, v. 25, p. 455-458.

labarthe-Hernández, G., Tristán, M. y Aranda-Gómez, J.J.,1982, Revisión estratigráfica del Cenozoico de la parte central del Estado de San Luis Potosí: Universidad Autónoma de San Luis Potosí, Instituto de Geología y Metalurgia, Folleto Técnico, v. $85,208 p$.

luhr, J.F., Aranda-Gómez, J.J. y Pier, J.G., 1989, Spinel- Iherzolitebearing, quaternary volcanic centers in San Luis Potosí, México,!. Geology, Mineralogy, and Petrology: Journal of Geophysical Research, v. 94, núm.B6, p. 7,916- 7,940.

luhr, J.F., Henry, C.D., Housh, T.B. y Aranda-Gómez, J.J. , 1998, Temporal changes in mafic magmatism in the Southern Basin and Range Province of México and West Texas: Eos Transactions American Geophysical Union, v. 79, n. 45, Fall Meet. Suppl., p. F945-F946.

luhr, J. F., Pier, J. G., Aranda-Gómez, J. J., Y Podosek, F., 1995, Crustal contamination in early Basin-and-Range hawaiites of the los Encinos volcanic field, central Mexico: Contributions to Mineralogyand Petrology, v. 118, p. 321-339.

Martínez-Serrano, R. G., Macías-Romo, C., Martiny-Kramer, B., Morán-Zenteno, D.J., Grajales-Nishimura, M. y Silva-Romo, G., 1996, Geocronología y características geoquímicas de la provincia volcánica del sur de México, transecto Huajuapan de león-Pinotepa Nacional, Oaxaca: GEOS, v. 16, n. 2, p.203-204.

McDowell, F.W., 1979, Ignimbrites of the Sierra Madre Occidental and their relation to the tectonic history of western Mexico: Geological. Society of America Special Paper, n. 180, p.113-124.
McDowell, F'w., Housh, T.B. y Wark, DA, 1999, Nature of the crust beneath west-central Chihuahua, Mexico, based upon $\mathrm{Sr}$, Nd, and $\mathrm{Pb}$ isotopic compositions at the Tom6chic volcanic center: Geological Society of America Bulletin, 111, 823-830.

McDowell, F.W. y Keizer, R.P., 1977, Timing of mid-Tertiary volcanism in the Sierra Madre Occidental between Durango City and Mazatlan, Mexico: Geological Society of America Bulletin, v. 88, p. 1,479-1,486.

McDowell, F.W. y Roldán-Ouintana, J., 1993, Geochronology of Mesozoic-Cenozoic magmatism in south-central Sonora: A progress report, in González, C. y Vega, I., eds., Ill Simposio de la geología de Sonora y áreas adyacentes: Universidad Nacional Autónoma de México, Instituto de Geología, p. 77-80.

McDowell, F. W., Roldán-Ouintana, J., y Amaya-Martínez, R., 1997, Interrelationship of sedimentary and volcanic deposits with Tertiary extension in Sonora, Mexico: Geological Society of America Bulletin, v.109, n. 10, p. 1,349-1,360

Mora-Álvarez, G. y McDowell, F. W., En prensa, Miocene volcanism emplaced during late subduction and early rifting in the Sierra Santa Ursula of Westem Sonora, Mexico: Geological Society of America Special Paper, n. 334.

Morán-Zenteno, D. J., Martiny, B., Tolson-Jones, G., Solís-Pichardo, G. Alba-Aldave, I., Hernández-Bernal, M.S., Macias-Romo, C.,Martínez-Serrano, R., Schaaf, P., Silva-Romo, G., 1999, Geocronología y características geoquímicas de las rocas magmáticas terciarias de la Sierra Madre del Sur, este volumen.

Natali, S. G., y Sbar, M. I., 1982, Seismicity in the epicentral region of the 1887 northeastem Sonora earthquake, Mexico: Bulletin of the Seismological Society of America, v. 72, p. 181-196.

Nieto-Samaniego, A. F., Alaniz-Álvarez, S. A., y labarthe-Hemández, G., 1997, la deformación post-laramídica en la parte sur de la Mesa Central, México: Revista Mexicana de Ciencias Geológicas, v. 14, p. 13-25.

Nieto-Samaniego, A. F., Ferrari, I., A1aniz-Álvarez, S. A., labartheHernández, G. y Rosas-Elguera, J. G., 1999, Variation of Cenozoic extension and volcanism across the southem Sierra Madre Occidental volcanic province, Mexico: Geological Society of America Bulletin, v. 111, p. 347-363.

Nimz, G.J., Cameron, K.I., Cameron, M., y Morris, S.I., 1986, The petrology of the lower crust and upper mantle beneath southeastem Chihuahua, Mexico: a progress report: Geofísica Internacional, v. 25, n.1, p. 85-116.

Nimz, G.J., Cameron, K.I. y Niemeyer, S., 1993, The Ia Olivina pyroxenite suite and the isotopic compositions of mantle basalts parental to mid-Cenozoid arc volcanism of northern Mexico: Journal of Geophysical Research, v. 98, n. B4, p. 6,489-6,509.

Nimz, G.J., Cameron, K.I. y Niemeyer, S., 1995, Formation of mantle lithosphere beneath northern Mexico: Chemical and Sr$\mathrm{Nd}-\mathrm{Pb}$ isotopic systematics of peridotite xenoliths from Ia Olivina: Journal of Geophysical Research, v. 100, n. B3, p. $4,181-4,196$

Ortega-Gutiérrez, F., Mitre-Salazar I. M, Roldán-Ouintana, J., Aranda-Gómez, J. J., Morán-Zenteno, D., Alaníz-Alvarez, S. A. y Nieto-Samaniego, A. F., 1992, Carta geológica de la República Mexicana (1:2,000.000): Universidad Nacional Autónoma México, Instituto de Geología, Secretaria de Energía, Minas e Industria Paraestatal, Consejo de Recursos Minerales, 1 mapa y texto explicativo.

Pantoja-Alor, J., 1983, Geocronometría del magmatismo CretácicoTerciario de la Sierra Madre del Sur: Boletín Sociedad Geológica Mexicana, v. XLIV, núm. 1, p. 1-20.

Paz-Moreno, F. A., Demant, A., 1998, los basaltos alcalinos del Mioceno tardío de la región de Saric (NO Sonora): Geología, mineralogía y geoquímica, in Alaniz-Álvarez, SA, Ferrari, I. y Nieto-Samaniego, A.F. eds., libro de Resumenes Primera Reunión Nacional de Ciencias de la Tierra, México, D.F., 
Sociedad Geológica Mexicana, p. 107.

Pier, J.G., Luhr, J.F., Podosek, FA y Aranda-Gómez, J.J., 1992, The La Breña-El Jagüey maar complex, Durango, Mexico: 11. Petrology and geochemistry, Bulletin 01 Volcanology, v.54, p. $405-428$.

Pier, J.G., Podosek, F., Luhr, J.F., Brannon, J. y Aranda-Gómez, J.J., 1989, Spinel-Iherzolite-bearing, quaternary volcanic centers in San Luis Potosi, México, 11. $\mathrm{Sr}$ and $\mathrm{Nd}$ isotopic systematics: Journal 01 Geophysical Research, v. 94, n. B6, p. $7,941-7,951$.

Rudnick, A. L., YCameron, K. L., 1991, Age diversity 01 the deep crust in northern Mexico: Geology, v. 19, p. 1,197-1,200.

Ruiz, J., Patchett, P. J. Y Arculus, R. J., 1988, Nd-Sr isotope composition 01 the lower crustal xenoliths -Evidence lor the origín 01 mid-Tertiary lelsic volcanics in Mexico: Contributions to Mineralogy and Petrology, v. 99, p. 36-42.

Ruiz, J., Patchett, P.J. y Arculus, A.J., 1990, Reply to "Comments on $\mathrm{Nd}-\mathrm{Sr}$ isotopic compositions 01 lower crustal xenoliths Evidence lor the origin 01 mid-Tertiary volcanics in Mexico", by K.L. Cameron and J.V. Robinson: Contributions to Mineralogy and Petrology, v. 104, p. 615-618.

Scholz C. , Barazangi M., Sbar M.L., 1971, Late Cenozoic evolution 01 the Great Basin, western United States, as an ensialic interarc basin: Geological Society 01 America Bulletin, v.82, p. 2,979-2,990

Severinghaus, J. y Atwater, T., 1990, Cenozoic geometry and thermal state 01 the subducting slabs beneath western North America, in Wernicke, B. P. ed. Basin and Range extensional tectonics near the latitude 01 Las Vegas, Nevada: Boulder, Colorado, The Geological Society 01 America, Memoir, n. 176, p. 1-22.

Schlische, A. W., 1995, Geometry and origin 01 lault-related lolds in extensional settings: American Association 01 Petroleoum Geologists Bulletin, v. 79, p. 1,661-1,678.

Smith, R.O., 1993, The Agua de Mayo mid-Cenozoic volcanic group and related xenoliths Irom La Olivina SE Chihuahua, Mexico: Santa Cruz, CA, University 01 Calilornia, Master 01 Science thesis, $112 \mathrm{p}$.

Smith, R. D., Cameron, K. L., McDowell, F. W., Niemeyer, S. y Sampson, D. E., 1996, Generation 01 voluminous silicic magmas and lormation 01 mid-Cenozoic crust beneath northcentral Mexico-evidence Irom ignimbrites, associated lavas, deep crustal granulites, and mantle pyroxenites: Contributions to Mineralogy and Petrology, v. 123, p. 375-389.

Stewart, J.H., 1998, Regional characteristics, tilt domains, and extensional history 01 the late Cenozoic Basin and Range province, westem North America, in Faulds, J.E y Stewart. J.H., eds., Accomodation zones and transfer zones: The regional segmentation 01 the Basin and Range Province: Boulder, Colorado, Geological Society 01 America, Special Paper, n. 323, p. 47-73.

Stewart, J. H., Y Roldán-Quintana, J.,1994, Map showing late Cenozoic extensional tUt pattems and associat00 structures in Sonora and adjacent areas, Mexico (1:1,000,000): U.S. Geological Survey, 1 mapa.

Stewart, J. H, Anderson, R.E., Aranda-Gómez, J.J., Beard, L.S., Bill ingsley, G.H., Cather, S.M., Dilles, J.H., Dokka, R.K., Faulds, J.E., Ferrari, L., Grose, T.L.T., Henry, C.D., Janeke, S.U., Miller, D.M., Richard, S.M., Rawley, P.D., RoldánQuintana, J., Scott, A.B., Sears, J.W. y Williams, V.S, 1998, Map showing Cenozoic tUt domains and associatOO structural leatures, westem North America, in Faulds, J. E. YStewart, J. H., OOs. Accomodation zonas and transler zones: The regional segmentation of the Basin and Range Province, Boulder, Colorado, Geological Society 01 America, Special Paper, n.323, Plate 1.

Stock, J. M., Y Hodges, K. V., 1989, Pre-Pliocene extension around the Gulf 01 Calilomia and the transfer 01 Baja Califomia to the Pacific Plate: Tectonics, v. 8, p. 99-115.

Swanson, EA., Keizer, R.P., Lyons, J.1. y Clabaugh, S.E., 1978, Tertiary volcanism and caldera development near Durango City, Sierra Madre Occidental, Mexico: Geological Society of America Bulletin, v. 89, p. 1,000-1,012.

Swanson, EA., y McDowell, FW., 1984, Calderas of the Sierra Madre Occidental volcanic field, Western Mexico: Journal of Geophysical Research, v. 89, n. B10, 8,787-8,799.

Torres-Hernández, J. R., 1998, Evidencias de magmatismo sintectónico en el campo volcánico de San Luis Potosí, S.L.P., México: GEOS, v. 18, p. 373.

Tristán-González, M., 1986, Estratigralía y tectónica del graben de Villa de Reyes, en los estados de San Luis Potosi y Guanajuato, Universidad Autónoma San Luis Potosí, InstiMo de Geología, Folleto Técnico 107, 91 p.

Wemicke, B. P., Christiansen, R. L., England, P.C. y Sonder, L.J., 1987, Tectonomagmatic evolution 01Cenozoic extension in the North American Cordillera, in Coward, M. P., Dewey, J. F. Y Hancock, L. eds. Continental Extensional Tectonics, Geological Society, Special Publication, núm.28, p. 203-221.

Yamamoto, J., 1993, Actividad microsísmica en el área de Canatlán, Durango y su relación con la geología regional: Geofísica Intemacional, v. 32, n. 3, p. 501-510. 